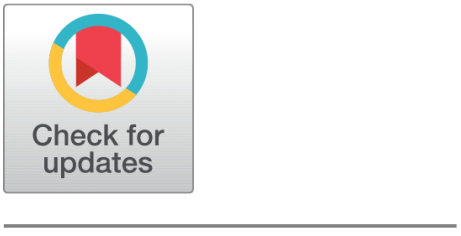

open ACCESS

Received: 08.04.2021

Accepted: 21.04 .2021

Published: 09.07.2021

Citation: Srinivasan R, Vivekanandan M (2021) On Packing Colouring of Transformation of Path, Cycle and Wheel Graphs. Indian Journal of Science and Technology 14(23): 1975-1981. https ://doi.org/10.17485/IJST/v14i23.606

* Corresponding author.

srinivasanmaths@gmail.com

Funding: None

Competing Interests: None

Copyright: @ 2021 Srinivasan \& Vivekanandan. This is an open access article distributed under the terms of the Creative Commons Attribution License, which permits unrestricted use, distribution, and reproduction in any medium, provided the original author and source are credited.

Published By Indian Society for Education and Environment (iSee)

ISSN

Print: 0974-6846

Electronic: 0974-5645

\section{On Packing Colouring of Transformation of Path, Cycle and Wheel Graphs}

\author{
R Srinivasan ${ }^{1 *}, \mathrm{M}$ Vivekanandan ${ }^{2}$ \\ 1 Department of Ancient Science, Faculty of Science, Tamil University, Thanjavur, 613006, \\ Tamil Nadu, India \\ 2 Department of Mathematics, Parisutham institute of technology and Science, Thanjavur, \\ 613006, Tamil Nadu, India
}

\section{Abstract}

Objectives: To compute the packing chromatic number of transformation of path graph, cycle graph and wheel graph. Methods: The packing chromatic number $\chi_{p c}(H)$ of a graph $H$ is the least integer $m$ in such a way that there is a mapping $C: V(H) \rightarrow(1,2, \ldots, m\}$ such that the distance between any two nodes of colour $k$ is greater than $k+1$. Findings: The packing chromatic number of the transformation of the graph $\chi_{p c}\left(H^{p q r}\right)$ where $p, q, r$ be variables which has the values either positive sign $(+)$ or a negative sign $(-)$ then $H^{p q r}$ is known as the transformation of the graph $H$ such that $V(H)$ and $E(H)$ belonging to the vertex set of $H^{p q r}$ and $\alpha(H), \beta(H)$ also belonging to $V(H), E(H)$ of the graph. Obtained the values of the packing chromatic number of transformation of path graph, cycle graph and wheel graph. Applications: Chromatic number applied in Register Allocations, a compiler is a computer program that translates one computer language into another. To improve the execution time of the resulting code, one of the techniques of compiler optimization is register allocation; if the graph can be colored with $\mathrm{k}$ colors then the variables can be stored in $\mathrm{k}$ registers.

Keywords: path graph; cycle graph; wheel graph; packing chromatic number

\section{Introduction}

Graph theory in mathematics means the study of graphs. Graphs are one of the major areas of Discrete Mathematics. Usually, a graph is referred to as the collection of nodes which is also called vertices and a collection of arcs which is called edges. Let $H=$ $(V(H), E(H))$ be a graph with $n$ vertices and $m$ edges, colouring the vertices of the graph $H$ such that any two adjacent vertices in the graph not sharing the same colour, now we are in a position to count the minimum requirement of colour to satisfying the condition that no two adjacent vertices have the same colour. Having coloured the vertices we can classify them into non-identical sets such that one set containing one colour and the other set containing another colour and so on. This is a partitioning problem. The colouring and partitioning can be done on vertices or edges of a graph.

Proper colouring is nothing but colouring all the vertices in which no adjacent vertices have the same colour. 
The intention of the packing chromatic number of a graph was established in ${ }^{(1)}$ by Goddard et al. There will be more difficult to identify the points in a mesh where two non-identical points are managing the unchanged frequency unless both the points are positioned not to very close. With this representation all points are positioned at nodes in the $i$ packing, $X_{i}$, are authorized to convey the uniform repetition only after distance $i$, this ideology has been developed a lot and used in many fields such as biological diversity, resource placements and so on.

Let $H=(V(H), E(H))$ be a graph and let $\mathrm{r}$ be any non-negative integer. Then $X$ be the subset of the vertex set of the graph $\mathrm{H}$, is an $\mathrm{r}$-packing provided the vertices of $\mathrm{X}$ are pairwise at a distance greater than the value of $r$. The uttermost number of $r$-packing in a graph $\mathrm{H}$ is called the $r$-packing number of $\mathrm{H}$ indicated by $\rho_{r}(H)$, this non-negative integer $r$ is nothing but the width of the packing $\mathrm{X}$.

Vizing $^{(1)}$ constituted that the determining the lower bounds for the Cartesian product of any two graphs. Williams \& Roy ${ }^{(2)}$ discussed the determination of packing chromatic number by joining two different graphs, in the name of comb graph, ladder graph, circular ladder graph and windmill graph. Brešar \& Zmazek ${ }^{(3)}$ induced on the independence graph of a graph and the independence number of the Cartesian product of graphs has been developed by Martin et al. ${ }^{(4)}$ and again this has been further developed by Klavžar in ${ }^{(5)}$ as some new bounds and exact results on the independent number of Cartesian product graphs. Derya et al. (6) discussed the packing chromatic number of transformation of graphs.

\section{Preliminary}

Definition 2.1 A simple digraph is called a path whose vertices can be linearly ordered so that there is an edge with tail $x$ and head $y$ iff $y$ immediately follows $x$ in the vertex order.

Definition 2.2 A cycle is defined similarly to the path provided ordering the vertices on a circle.

Definition 2.3 A wheel graph $W_{n}$ of order $\mathrm{n}$ is a graph that contains a cycle of order $\mathrm{n}$-1and every vertex of the cycle is connected to one other vertex of the cycle.

Definition 2.4. A proper $k$-vertex colouring of a simple graph $H=(V, E)$ is defined as vertex colouring from a set of $k$ colours such that no two adjacent vertices share the same colour.

$C: V(H) \rightarrow\left(c_{1}, c_{2}, \ldots, c_{k}\right\}$ Such that $\forall u, v \in E(H) / C(u) \neq C(v)$.

Definition $2.5^{(7)}$ Let $\mathrm{H}$ be any graph and we need to find the smallest integer $l$ in such a way that the vertex set of the graph $\mathrm{H}, V(H)$ can be partitioned into $l$ packings as $X_{1}, X_{2}, \ldots, X_{l}$ with pairwise, unlike widths. This split-up has packings of $l$ inconsistent widths and the aim is to minimize $l$, the non-negative integer $l$ meant as the packing chromatic number of $H$ and marked it by $\chi_{p c}(H)$.

Definition $2.6^{(8)}$ Let $\mathrm{H}$ be any graph and $p, q, r$ be variables which have the values either positive sign $(+)$ or a negative sign () then $H^{p q r}$ is known as the transformation of the graph $H$ such that $V(H)$ and $E(H)$ belonging to the vertex set of $H^{p q r}$ and $\alpha(H), \beta(H)$ also belonging to $V(H), E(H)$

Definition 2.7 The maximal number of independent vertices, that is no two of the vertices have an edge in common which is denoted as $\alpha(H)$ and the minimal number of covering vertices, that is edges from these vertices cover all the edges which is referred as $\beta(H)$.

Proposition $2 . \mathbf{8}^{(9)}$ If - , then $\chi_{p c}(H) \leq \beta(H)+1$

Theorem 2.9 ${ }^{(6)}$ Let $H$ be a graph of order $n$ then $\alpha(H)+\beta(H)=1$

In this paper, Section 1, we have given the introduction to graph colouring. In Section 2 the preliminary definitions and theorems have given. In Section 3, we discuss the packing chromatic number of transformation of path graph, in Section 4 we scrutinize about the packing chromatic number of transformation of cycle graph and in Section 4, we study about the packing chromatic number of transformation of wheel graph.

\section{The packing chromatic number of transformation of the path graph}

In this section, we discuss the packing chromatic number of transformation of the path graph

Theorem $3.1 \chi_{p c}\left(P_{m}^{---}\right)=2 m-3$

Proof. Let $P_{m}$ be the path graph with $m$ vertices, $P_{m}^{---}$be the transformation graph of $P_{m}$ with order $2 m-1$.

Let $u_{1}, u_{2}, u_{3}, \ldots, u_{2 m-1}$ be the vertices of the graph as shown in the following figure, where $u_{1}, u_{3}, u_{5}, \ldots, u_{2 m-1}$ be the vertices of the path graph $P_{m}$ and $u_{2}, u_{4}, u_{6}, \ldots, u_{2 m-2}$ be the edges of the path graph $P_{m}$. By transforming the graph $P_{m}$, we obtain $P_{m}^{---}$. 

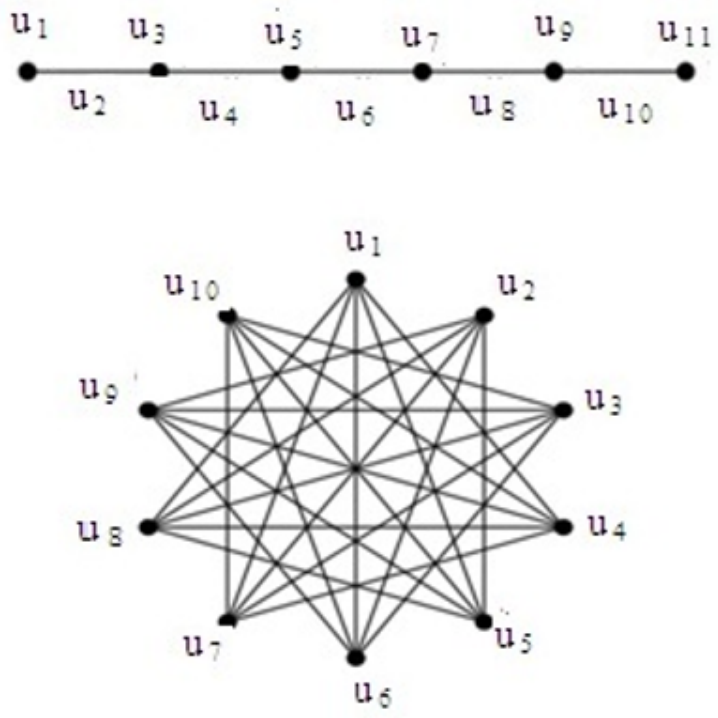

Fig 1.

By the above figure, we can have $\alpha\left(P_{m}^{---}\right)=3$

Let us consider the following cases,

Case (i). Let $u_{1} \& u_{2 m-1}$ be the end vertices of $P_{m}$ which is belonging to the vertices set of $P_{m}^{---}$and $u_{2} \& u_{2 m-2}$ be the pendent edges of $P_{m}$ which is belonging to vertices set of $P_{m}^{---}$

$$
\begin{aligned}
& d\left(u_{1}, u_{2}\right)=2 \\
& d\left(u_{1}, u_{3}\right)=2
\end{aligned}
$$

Then the distance between the vertices $u_{1}$ and the rest of all the vertices of $P_{m}^{---}$is 1 .

$$
\begin{gathered}
d\left(u_{1}, u_{4}\right)=1 \\
d\left(u_{1}, u_{5}\right)=1 \\
\vdots \\
d\left(u_{1}, u_{2 m-1}\right)=1
\end{gathered}
$$

By the same way,

$$
\begin{aligned}
& d\left(u_{2}, u_{3}\right)=2 \\
& d\left(u_{2}, u_{4}\right)=2
\end{aligned}
$$

Then the distance between the vertices $u_{2}$ and the rest of all the vertices of $P_{m}^{---}$is 1 .

$$
\begin{gathered}
d\left(u_{2}, u_{5}\right)=1 \\
d\left(u_{2}, u_{6}\right)=1 \\
\vdots \\
d\left(u_{2}, u_{2 m-1}\right)=1
\end{gathered}
$$


And also,

$$
\begin{aligned}
& d\left(u_{2 m-2}, u_{1}\right)=1 \\
& d\left(u_{2 m-2}, u_{2}\right)=1 \\
& \vdots \\
& d\left(u_{2 m-2}, u_{2 m-5}\right)=1 \\
& d\left(u_{2 m-2}, u_{2 m-4}\right)=2 \\
& d\left(u_{2 m-2}, u_{2 m-3}\right)=2 \\
& d\left(u_{2 m-2}, u_{2 m-1}\right)=2
\end{aligned}
$$

Case (ii). Let $u_{3}, u_{4}, \ldots, u_{2 m-4,} u_{2 m-3} \in V\left(P_{m}^{---}\right)$

$$
\begin{aligned}
& d\left(u_{3}, u_{4}\right)=2 \\
& d\left(u_{3}, u_{5}\right)=2
\end{aligned}
$$

Then the distance between the vertices $u_{3}$ and the rest of all the vertices of $P_{m}^{---}$is 1.

$$
\begin{aligned}
& d\left(u_{3}, u_{6}\right)=1 \\
& d\left(u_{3}, u_{7}\right)=1 \\
& \vdots \\
& d\left(u_{3}, u_{2 m-1}\right)=1
\end{aligned}
$$

Now consider $u_{4}, u_{6}, \ldots, u_{2 m-4}, \in\left(P_{m}^{---}\right)$

$$
\begin{aligned}
& d\left(u_{4}, u_{5}\right)=2 \\
& d\left(u_{4}, u_{6}\right)=2
\end{aligned}
$$

Then the distance between the vertices $u_{4}$ and the rest of all the vertices of $P_{m}^{---}$is 1 .

$$
\begin{aligned}
& d\left(u_{4}, u_{7}\right)=1 \\
& d\left(u_{4}, u_{8}\right)=1 \\
& \vdots \\
& d\left(u_{4}, u_{2 m-1}\right)=1
\end{aligned}
$$

From the above, $\operatorname{dim}\left(P_{m}^{---}\right)=2$, then other than this colour class we can have one component, so, $\chi_{p c}\left(P_{m}^{---}\right) \leq 2 m-4+1$. Therefore $\chi_{p c}\left(P_{m}^{---}\right) \leq 2 m-3$.

Let $\chi_{p c}\left(P_{m}^{---}\right)=2 m-4$. We know that $\alpha\left(P_{m}^{---}\right)=3$, then colour 1 is assigned to three vertices of $P_{m}^{---}$. Also, we know that - , then the remaining vertices must be assigned with other than the colour already assigned. Hence the required colours for making the packing colouring $P_{m}^{---}$is $2 m-4+1$

Therefore $\chi_{p c}\left(P_{m}^{---}\right)=2 m-3$.

Corollary: $3.2 \chi_{p c}\left(P_{6}^{---}\right)=9$

The following figure is a transformation of the graph $P_{6}$

It is visible that a minimum of 9 colours required for packing colouring for the graph $P_{6}^{---}$

Hence $\chi_{p c}\left(P_{6}^{---}\right)=9$. 


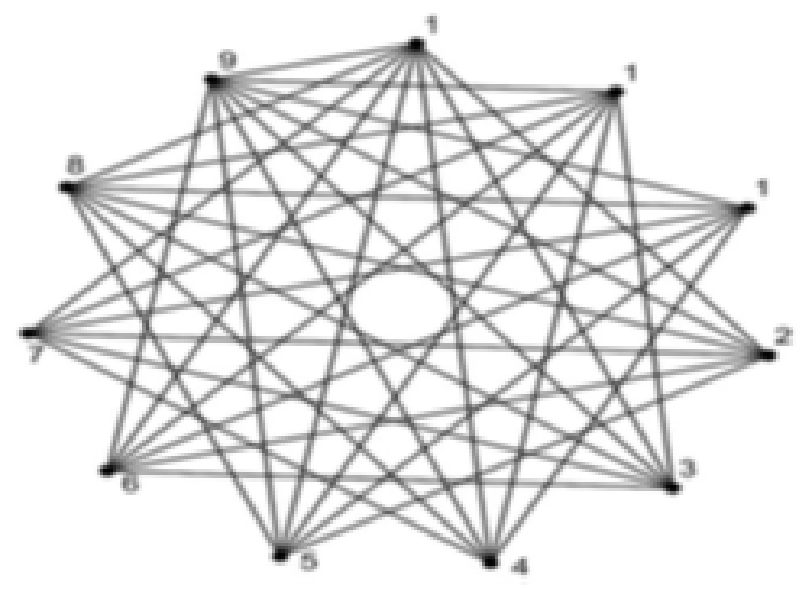

Fig 2.

\section{The packing chromatic number of transformation of the cycle graph}

In this section, we discuss the packing chromatic number of transformation of the cycle graph.

Theorem $4.1 \chi_{p c}\left(C_{m}^{---}\right)=2 m-2$ where $m>3$.

Proof. Let $C_{m}$ be the cycle graph with $m$ vertices provided $m$ is greater than 3 . Then $C_{m}^{---}$be the transformation of the graph $C_{m}$ with order $2 m$.

Let $u_{1}, u_{2}, u_{3}, \ldots, u_{m}$ be the vertices of $C_{m}$ and $u_{m+1}, u_{m+2}, \ldots, u_{2 m}$ be the edges of $C_{m}$, then by the definition of the transformation of the graph, $u_{1}, u_{2}, u_{3}, \ldots, u_{2 m}$ be the vertices of the graph $C_{m}^{---}$

Let us allocate colour $C_{1}$ from the colour class $X_{1}$ to the vertex $u_{1}$. The vertices apart from $u_{1}$, in which the distance between the vertices $u_{1} \& u_{i}$ is greater than 1 , can be allocated with other colours.

Consider the three vertices say, $u_{1}, u_{m+1}, u_{2}$, as $\alpha\left(C_{m}^{---}\right)=3$, colour $C_{1}$ can be allocated to these vertices. Also, we know that $\operatorname{dim}\left(C_{m}^{---}\right)=2$ and $d\left(u_{3}, u_{i}\right)$ is greater than 2 , colour $C_{1}$ is not possible to allocate, so we can choose another colour. Hence $\chi_{p c}\left(C_{m}^{---}\right) \leq 2 m-2$.

Now let us assume that $\chi_{p c}\left(C_{m}^{---}\right) \leq 2 m-3$, then by the same argument of the theorem 3.1, we may get the result as $\chi_{p c}\left(C_{m}^{---}\right) \geq 2 m-2$

Therefore $\chi_{p c}\left(C_{m}^{---}\right)=2 m-2$.

Corollary: $4.2 \chi_{p c}\left(C_{5}^{---}\right)=8$

The following figure is a transformation of the graph $C_{5}$

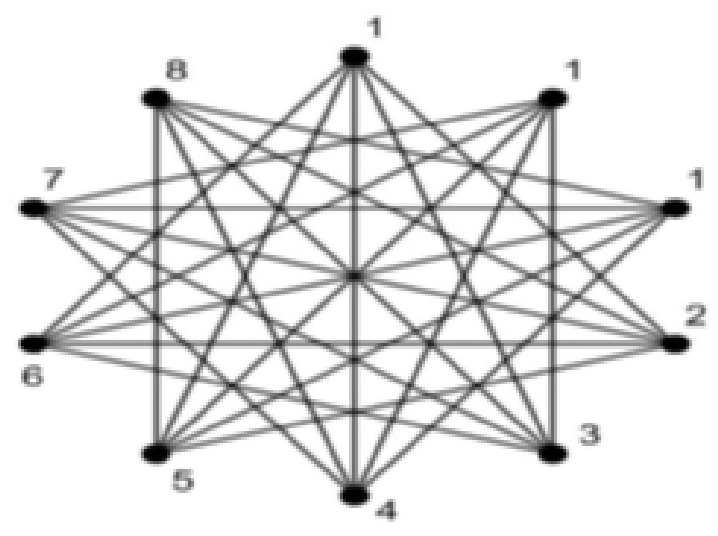

Fig 3.

It is visible that a minimum of 8 colours required for packing colouring for the graph $C_{5}^{---}$

Hence $\chi_{p c}\left(C_{5}^{---}\right)=8$. 


\section{The packing chromatic number of transformation of the wheel graph}

In this section, we discuss the packing chromatic number of transformation of the cycle graph

Theorem $5.1 \chi_{p c}\left(W_{m}^{---}\right)=3 m-4$ where $m \geq 5$.

Proof. Let $W_{m}$ be the wheel graph with $m$ vertices provided $m$ is at least 5 . Then $W_{m}^{---}$be the transformation of the graph $W_{m}$ with order $3 m-2$.

Let $u_{1}, u_{2}, u_{3}, \ldots, u_{m}$ be the vertices of $W_{m}$ and $u_{m+1}, u_{m+2}, \ldots, u_{3 m-2}$ be the edges of $C_{m}$, then by the definition of the transformation of the graph, $u_{1}, u_{2}, u_{3}, \ldots, u_{3 m-2}$ be the vertices of the graph $W_{m}^{---}$

By the same arguments of the above theorems 3.1 and 4.1 , we can prove this result as $\chi_{p c}\left(W_{m}^{---}\right)=3 m-4$

Hence the theorem.

Corollary: $5.2 \chi_{p c}\left(W_{5}^{---}\right)=11$

The following figure is a transformation of the graph $W_{5}$

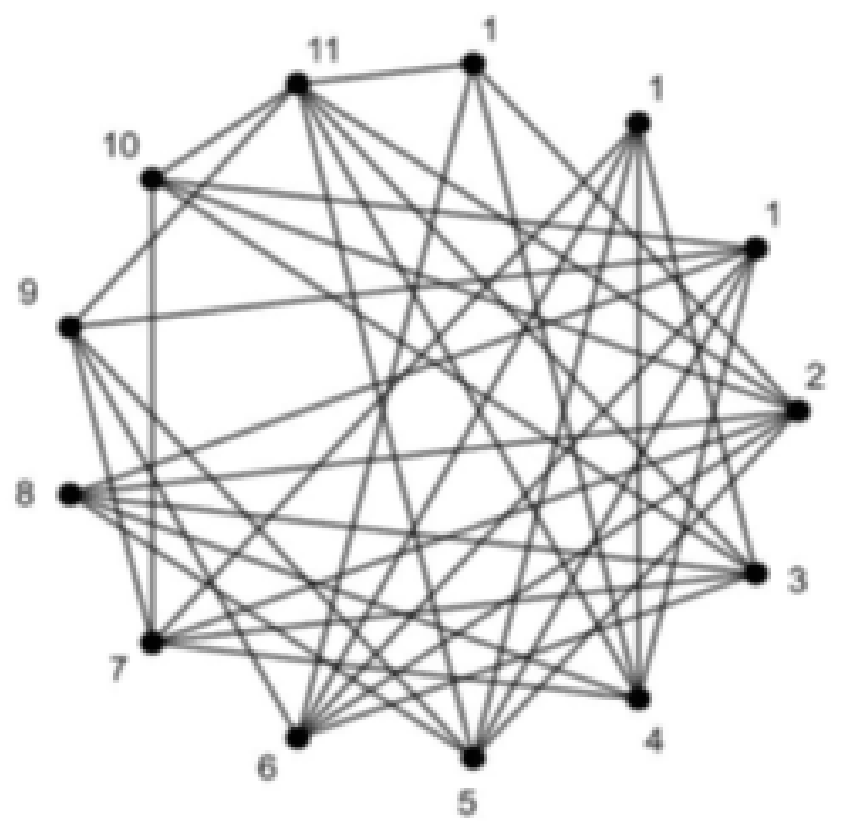

Fig 4.

It is visible that a minimum of 8 colours required for packing colouring for the graph $W_{5}^{---}$

Hence $\chi_{p c}\left(W_{5}^{---}\right)=11$.

\section{Conclusion}

In this paper, we enumerate the packing chromatic number of transformation of path graph, the packing chromatic number of transformation of cycle graph and derived the packing chromatic number of transformation of wheel graph using the definition of transformation of graph. In particularly we have shown each section with an example and verified the derived result.

The packing chromatic number for the Cartesian product of the transformation of graphs is under investigation. In future, we propose the packing chromatic number of transformation of Cartesian product of graphs. And packing chromatic number of transformation of wheel related graphs is under investigation.

\section{References}

1) Vizing VG. Cartesian product of graphs. Vychisl Sistemy. 1963;9:30-43. Available from: 10.4236/ojdm.2015.54008.

2) William A, Roy S. Packing Chromatic numbers of Certain Graphs. International Journal of Pure and Apllied Mathematics. 2013;87(6):731-739. Available from: https://dx.doi.org/10.12732/ijpam.v87i6.1.

3) Brešar B, Zmazek B. On the independence graph of a graph. Discrete Mathematics. 2003;272(2-3):263-268. Available from: https://dx.doi.org/10.1016/ s0012-365x(03)00194-8. 
4) Martin SP, Powell JS, Rall DF. On the independence number of the Cartesian product of caterpillars. Ars Combin. 2001;60:73-84. Available from: https://www.sciencedirect.com/science/article/abs/pii/S1571065307001205.

5) Klavžar S. Some new bounds and exact results on the independence number of Cartesian product graphs. Ars Combin. 2005;74:173-186. Available from: https://www.fmf.uni-lj.si/ klavzar/preprints/INDEP1.pdf.

6) Durgun DD, Dortok HBO. Packing chromatic number of transformation graphs. Thermal science. 2019;23(6):S1991-S1995. Available from: http: //www.doiserbia.nb.rs/img/doi/0354-9836/2019/0354-98361900363D.pdf.

7) Brešar B, Klavžar S, Rall DF. On the packing chromatic number of Cartesian products, hexagonal lattice, and trees. Discrete Applied Mathematics. 2007;155(17):2303-2311. Available from: https://dx.doi.org/10.1016/j.dam.2007.06.008.

8) Božović D, Peterin I. A note on the packing chromatic number of lexicographic products. Discrete Applied Mathematics. 2021;293:34-37. Available from: https://dx.doi.org/10.1016/j.dam.2021.01.010.

9) Baoyindureng W, Jixiang M. Basic Properties of Total Transformation Graphs. Journal of Mathematical Study. 2001;34. 